\title{
Distributed Recharging Rate Control for Energy Demand Management of Electric Vehicles
}

\author{
Q. R. Hamid Student Member, IEEE, and J. A. Barria Member, IEEE,
}

\begin{abstract}
In this paper we propose a distributed recharging rate control algorithm which combines the objectives of regulating frequency and improving the utilization of electric generators. An incentive policy $\kappa$ is created that encourages Electric Vehicles (EVs) to demand energy when non-EV demand is low and the electric generators are underutilized. EVs also act as frequency regulators which can control their participation role by modifying their respective payment rate $w_{i}$. The proposed distributed recharging rate control algorithm can realize a Demand Management solution for EVs and does not require explicit real time communication from the electric generators or between the recharging sockets. A mechanism is presented that can trade off an ideal incentive policy with its approximation, and enable the integration of the proposed controller with legacy protection system. Simulation is used to assess the algorithm and to highlight its embedded characteristics.
\end{abstract}

Index Terms-Demand management, distributed algorithms, frequency regulation, electric vehicles, multi-agent systems.

\section{INTRODUCTION}

A CCORDING to the International Energy Agency's $\mathrm{CO}_{2}$ emission report 2009 [1], transport is a significant contributor to global $\mathrm{CO}_{2}$ emission with $23 \%$ share. As expected, in the transport industry, a transition to Electric Vehicles (EVs) is taking place and it is very likely that most transport in the future will be electric [2]. Several studies have attempted to predict the impacts of EVs on the electric grid [3]. It is well understood that without any intervention from the electric grid EVs will add to evening peak demand as each EV would recharge as soon as it is plugged in. With increasing penetration of renewable energy sources, this scenario will not only cause operational problems for the electric grid but will also further aggravate the problem of underutilization of electric generators resulting from large peak demand and reduced off peak demand. Various solutions that cope with intermittent renewable energy sources have been suggested in the literature e.g., operating large electric generators as reserves has been suggested in [4], storage of energy is considered in [5] and the use of Demand Side Management (DSM) is addressed in [6]. Recently, there has been an increased interest in using $\mathrm{EV}$ batteries as part of storage systems to counterbalance the intermittency of renewable energy sources. Ideally, storage systems should be managed in such a way that transport and power system are integrated [7], [8]. We refer to these systems as EV energy Demand Management (DM) systems.

Kempton et al. [9] suggest that EVs may be used beneficially in Vehicle-to-Grid (V2G) regime where EVs act as small

The authors are with the Department of Electrical and Electronic Engineering at Imperial College London. Phone : +44-(0)20-759 46275, Fax +44-(0)20-759 46274 e-mail: j.barria@imperial.ac.uk generators to absorb some of the peak demand. Kempton et al. [6] and Lund et al. [8] have also suggested that EVs may be used to support renewable energy sources if their demand is managed intelligently. Lopes et al. [10] present similar suggestions and compare three recharging strategies namely $i$ ) dumb recharging $i$ ) dual tariff and iii) smart recharging. White et al. [11] address the use of V2G mode of operations and suggest a dual functionality for EVs as frequency regulators and peak shavers. They showed that there are strong financial incentives for EV owners to use EVs for frequency regulation. Finally, using DM, EVs may also be integrated with the power system as a subset of DSM as suggested in [6], [8] and [10]. While these works highlight the importance of EV energy DM, they hardly expand on suggesting how such a DM system may be implemented.

In this paper we propose a recharging rate control algorithm for parked EVs, where a large percentage of these EVs behave as variable power and delay tolerant loads and coexist with other types of loads. The proposed recharging rate control algorithm $i$ ) can realize a DM solution to reconcile energy demand from autonomous EVs with the output of $e . g$., renewable energy sources and $i$ ) can realize an alternate mechanism for frequency regulation in the event of $e . g$., changes in output power of electric generators.

This paper is organized as follows: Section II presents a review of the related work. Section III describes the architecture of system under consideration, the components of system, and their inter relationship. Section IV presents a distributed recharging rate control algorithm for recharging EV batteries. Section $\mathrm{V}$ provides an implementation of recharging rate control and simulation results. Section VI uses an example to relate the recharging rate control with the recharging strategy of EVs and generation capacity control. We conclude this paper in Section VII and comment on future work.

\section{RELATED WORK}

An EV energy Demand Management problem is essentially a scheduling problem which manifests two key features: $i$ ) Each EV battery is recharged to the desired state of battery charge during the period between two consecutive journeys; $i$ i) The aggregate demand from EVs could fill the valley that nonEV demand produces. Feature ii) can also be interpreted to be aiming at minimizing the difference between the instantaneous marginal cost of generation and its average over 24 hours. Such interpretation includes the availability of renewable energy sources and hence the aggregate demand from EVs may not necessarily be seen as a flat valley filling demand.

The work on EV energy DM can be broadly classified into two distinct categories depending on the level of autonomy of 
EVs: $i)$ the recharging schedule is controlled by the electric grid $\mathrm{ii}$ ) the recharging schedule control is delegated to autonomous EV agents. In the first case EVs report their recharge requirements to a dispatch center which in turn produces a recharging schedule for each EV [12]. Despite the apparent simplicity of the electric grid controlled scheduling, to the best of our knowledge, polynomial time algorithms that compute optimal recharging schedules have not been published. In the second case each EV is allowed to self-schedule the recharging time according to the criterion known only to the respective EV agent [7], [13].

The research work on DM with autonomous EVs can be further divided into two classes: $i$ ) day ahead negotiation and ii) real time bargaining. The former class includes works where EVs can bid for energy the day before the actual demand [14]. The algorithm developed in this paper belongs to the latter class where EV agents bargain in real time and learn from historic demand patterns. The publications relevant to the work presented in this paper are reviewed in the remainder of this section.

Galus et al. [7] present a framework for recharging EVs using an energy hub system. They note that EVs must be granted autonomy in recharging decisions and suggest use of a multi-agent system (MAS). In principle, we support the use of a MAS framework for EV energy DM and pursue a similar broader objective of integrating transport and power systems. However, our solution is quite different: Galus et al. [7] integrate EVs into multi carrier energy networks by solving an optimal energy dispatch problem whereas in this paper we propose to integrate EVs as frequency regulators. Also, in [7] a hub manager aggregates several hundred EVs and requires information from each $\mathrm{EV}$ to provide a solution. The hub manager maintains a list of arriving/departing EVs and allows new EVs to join only at the start of 15 minute intervals. The solution proposed in this paper works with or without aggregation of EVs and allows EVs to join at arbitrary times.

Though the economic aspects of DM can be understood using classical economics and game theory, the mechanics of resulting energy transactions and the impacts on the operation of the system have received little attention in the literature. For example, Vytelingum et al. [15] and Wei et al. [16] attempt to present MAS based solution to manage micro storage devices including EVs. However, they do not consider the actual mechanism by which agents acquire energy and assume that agents can buy it at market price. Such MAS can be integrated on top of the algorithm described in this paper.

Ma et al. [13] use non-cooperative game theory to analyze the recharging strategies of EVs and make the observation that the recharging games for EVs are conceptually similar to the routing games in networks. They consider EVs as cost minimizing rational agents coupled through a common energy price where each agent solves its local optimization problem. In the limit of infinite population, the decentralized strategies of EVs result in a unique Nash equilibrium that has the property of filling valleys in non-EV demand. In comparison to [13], our work addresses a few novel aspects of decentralized recharging of EVs. We show not only that EVs can decide strategy in a decentralized manner, but also that the impact of the strategy and resulting incentive can also be computed using decentralized recharging rate controllers. This paper presents a novel attempt to include in a unified framework: the recharging strategies of EVs, operational aspect of frequency regulation and the utilization of the electric generators.

Some studies on Load Frequency Control (LFC) or frequency regulation can be found in the literature [17]. Recently, researchers have become interested in using EVs to provide frequency regulation services [18]. The recharging rate control proposed in this paper may also be classified as an LFC mechanism. However, the mechanism suggested here is novel in that it combines frequency regulation with incentive policy provision for autonomous EV agents.

\section{System ARCHitecture}

\section{A. Physical Architecture}

Electric Vehicles connect to the distribution system through recharging sockets. Each recharging socket has its own recharging rate controller (socket agents). In the proposed architecture, recharging sockets are enabled round the clock and do not adhere to a centralized recharging policy. Each EV has an agent that can communicate with a recharging socket and make a demand for energy. Recharging sockets receive these demands and provide energy accordingly. Thus EVs may be considered as autonomous buyers and the recharging sockets as points of sale.

After completing a journey EVs will connect to recharging sockets. The ith EV agent will send a signal $w_{i, 1}[\$ / \mathrm{h}]$ to the socket agent indicating its preferred rate of payment to get recharged, and in return the socket agent will allocate a recharging rate of $p_{i}[\mathrm{kw}]$ and will broadcast on the current policy $1 / \kappa[\$ / \mathrm{kwh}]$. From now on we will also refer to $p_{i}[\mathrm{kw}]$ as the recharging rate of ith EV. EVs may also indicate their willingness to discharge in $\mathrm{V} 2 \mathrm{G}$ mode and communicate $w_{i, 2}$ [\$/h] to recharging sockets which will indicate their desired rate of payment for discharging.

When connecting to the recharging socket an EV agent may decide a value of $w_{i, 1}[\$ / \mathrm{h}]$ based on its current status and requirements for the next journey, which may include; current battery state of charge, expected remaining time to a new journey $\left(T_{L_{i}}\right)$, expected travel time of next journey and total budget for recharging $(B d)$.

\section{B. Logical Architecture}

The framework aims at exploiting the following characteristics of EVs to manage their demand.

1) EVs are indifferent to the exact time of recharge completion as long as usual/scheduled journeys are not affected.

2) EV population has heterogeneous energy needs.

- Not all EVs need to recharge their battery to maximum capacity each day.

- Not all EVs have the same journey patterns

3) Many EVs can tolerate uncertainty in exact battery state of charge provided they can achieve a minimum battery state of charge. 


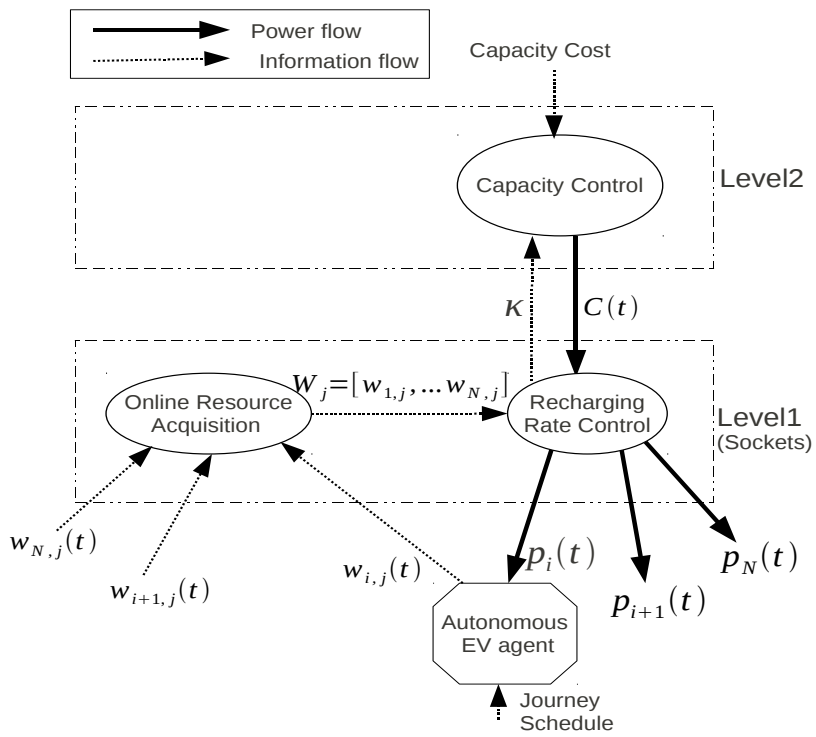

Fig. 1. Components of the Demand Management problem.

We decompose the EV energy DM problem into three inter related components, namely:

1) Distributed recharging rate control,

2) On line resource acquisition,

3) Capacity control.

Figure 1 shows the relationship between these components which are organized at two levels. Level 1 represents the activities on the demand side and level 2 represents activity on the generation side. We now briefly describe the function of each component.

Component 1: Distributed Recharging Rate Control: Component 1 controls the energy transfer rate for EVs based on real time availability of energy. Its aim is to balance the aggregate demand with the time varying generation capacity set by Component 3 . In the event that non-vehicular demand exceeds the generation capacity, Component 1 reduces the recharging rate for all EVs to zero and possibly allocates negative recharging rates (V2G mode of operations [9]). If generation capacity exceeds the non-vehicular demand, then recharging rates are allocated to EVs according to their respective payment rate $w_{i, 1}[\$ / \mathbf{h}]$. This paper will mainly focus on the solution of Component 1 of EV energy DM system.

Component 2: On line Resource Acquisition: This component is intended to capture the behaviour of autonomous EV agents when they submit $w_{i, 1}$, and to analyze the equilibrium properties of the system. Given a generation capacity, it is possible for an autonomous EV agent to increase its recharging rate $w_{i, 1}$ relative to other EVs. Increasing $w_{i, 1}$, however, does not mean that the recharging rate of that EV will necessarily increase because competing EVs agents can also decide to increase their respective $w_{k, 1}$. This situation can be modelled as a game [19] and in Section VI we present a simple instance of such game.

Component 3: Capacity Control: Component 1 uses capacity as an exogenous variable and cannot control it. A scenario may be constructed where capacity is zero and Component 1 can only reach one solution which is to reduce the recharging rate to zero for all EVs. Component 1 will enforce this solution irrespective of what EVs wish to pay. Hence, to ensure that EVs may receive sufficient power, decision on the generation side is needed, which is the capacity control. This capacity control problem can be modelled as a Revenue Management (RM) problem.

\section{Remarks on EV agents and Recharging Sockets}

In regards to the interaction between the EV and socket agents, upon connection the EV agent can submit to the socket agent an arbitrary small value of $w_{i, 1}[\$ / \mathrm{h}]$ which is the payment rate at which EV is willing pay for recharging. In turn, the EV agent obtains from the socket agent a signal $1 / \kappa$ [\$/kwh] which can be interpreted as the instantaneous price of unit of energy. In this setting the EV agent's action of submitting a $w_{i, 1}[\$ / \mathrm{h}]$ is equivalent to submitting a demand for $p_{i}=\kappa * w_{i, 1}[\mathrm{kw}]$. After this initial interchange of signals EV agents can continuously monitor changes in $1 / \kappa[\$ / k w h]$, and they can decide autonomously if their rate of payment $w_{i}[\$ / \mathrm{h}]$ needs modification. We assume that $\mathrm{EV}$ agents are continuously trying to reconcile their internal goals, as for example, their predicted battery state of charge $(B S O C)$ at the start of their next journey with their remaining budget and current payment rate.

On the other hand, the sockets are continuously solving a Nash bargaining problem that will allocate to each EV attached to the socket a recharging rate that is proportionally fair. At equilibrium, $1 / \kappa[\$ / \mathrm{kwh}]$ should be such that no EV changes its payment rate $w_{i, 1}[\$ / \mathrm{h}]$. Note that with the above mechanism EVs need only to communicate to the local socket $w_{i, 1}[\$ / \mathrm{h}]$ and can discover their equilibrium charging rates $p_{i}[\mathrm{kw}]$ in a distributed manner. We further note that from the perspective of a socket, the equilibrium payment rates $w_{i, 1}[\$ / \mathrm{h}]$ are still exogenous variables and can be considered arbitrary.

There are unlimited ways in which EV agents and socket agents can agree to more elaborated ways of interchanging information. For example, the socket agents could broadcast forecasts of near future $1 / \kappa[\$ / \mathrm{kwh}]$ values. This line of research is out of the scope of this paper but is certainly worth further investigation.

\section{Distributed Recharging Rate Control}

\section{A. Problem Formulation}

\section{Notation}

$V(t)=$ set of EVs recharging at time $t$,

$V_{V 2 G}(t)=$ set of EVs willing to participate in $\mathrm{V} 2 \mathrm{G}$ when needed,

$N=$ Number of recharging EVs,

$p_{i}=$ Recharging rate (at which energy is accumulat-

ing in the ith EV battery [kw] (or otherwise, when not specified, [p.u.]) ${ }^{1}$,

$w_{i, 1}=$ ith EV's payment rate (paid by EV) for recharging $[\$ / \mathrm{h}]$,

\footnotetext{
${ }^{1}$ p.u $=$ per unit. Hence, these units have been normalized
} 
$w_{i, 2}=$ ith EVs payment rate (paid to $\mathrm{EV}$ ) for discharging in $\mathrm{V} 2 \mathrm{G}$ mode $[\$ / \mathrm{h}]$,

$P_{S C H}(t)=$ Scheduled Power [p.u],

$P_{N E V}(t)=$ Power demand from non-EV loads [p.u.],

$C(t)=P_{S C H}(t)-P_{N E V}(t)=$ Net power capacity available to recharge EVs in G2V mode [p.u.],

$P_{V 2 G}(t)=$ Aggregate power drawn from EVs in $\mathrm{V} 2 \mathrm{G}$ mode [p.u.],

$p_{\text {imax }}=$ Maximum allowed power at which ith EV's battery can be recharged [p.u.],

$p_{\text {imax } V 2 G}=$ The maximum allowed power at which ith EV's battery can be discharged [p.u.],

$\Delta \omega=$ frequency deviation [p.u.],

$P_{E V}=\sum_{i=1}^{N} p_{i}$ [p.u.],

$\mathbf{P}=\left[p_{1}, p_{2}, \ldots p_{N}\right]$ [p.u.],

$W_{1}=\left[w_{1,1}, w_{2,1}, \ldots w_{N, 1}\right][\$ / \mathbf{h}]$.

Recharging sockets may advertise two modes of operation for EVs:

- Mode 1: if $C(t)>0$, The EVs in $V(t)$ act as energy sinks.

- Mode 2: if $C(t)<0$, EVs in $V_{V 2 G}(t)$ act as energy sources.

In addition to the EVs considered in sets $V(t)$ and $V_{V 2 G}(t)$, some EVs may choose to discharge their batteries while $C(t)>0$. In the framework here presented, we can add their discharging rates to $C(t)$ and consider them as virtual generators maximizing their revenue. Similarly, EVs are allowed to recharge when $C(t)<0$. If this is the case, they are assimilated as non-EV loads and their recharging rates are added to $P_{N E V}$.

We now introduce the two objective functions of the two optimization problems that the socket agents are continuously solving. The choice of these objective functions has its foundations in the Nash bargaining mechanism from cooperative game theory [20], [21]. The Nash bargaining solution framework enables the implementation of fair allocation of resources among contending agents and it can be seen as a generalization of the widely studied proportional fairness principle. For example, in [22] the proportional fairness principle was used to assign rates fairly to different contending elastic data traffic demands. For more on fairness and optimization the reader is referred to [23], [24]. With this choice of objective function in mind, the socket controllers (agents) set the recharging rate of connected EVs by solving the following optimization problems.

$\underline{\text { Mode } 1}$

$$
\begin{array}{ll}
\underset{\mathbf{P}}{\operatorname{maximize}} & \sum_{i \mid v_{i} \in V(t)} w_{i, 1}(t) \log \left(p_{i}(t)\right) \\
\text { subject to } & \sum_{i \mid v_{i} \in V(t)} p_{i}(t) \leq C(t) \\
& 0 \leq p_{i}(t) \leq p_{\text {imax }} \quad \forall i \mid v_{i} \in V(t)
\end{array}
$$

\section{Mode 2}

$$
\begin{array}{ll}
\underset{\mathbf{P}}{\operatorname{maximize}} & \sum_{i \mid v_{i} \in V_{V 2 G}(t)} w_{i, 2}(t) \log \left(-p_{i}(t)\right) \\
\text { subject to } & \sum_{i \mid v_{i} \in V_{V 2 G}(t)}-p_{i}(t) \leq P_{V 2 G}(t) \\
& -p_{\text {imax } V 2 G} \leq p_{i}(t) \leq 0 \quad \forall i \mid v_{i} \in V_{V 2 G}
\end{array}
$$

Note that for the proposed recharging rate control $w_{i, 1}(t)$, $w_{i, 2}(t), C(t)$ and $P_{V 2 G}(t)$ are exogenous variables and cannot be controlled: $w_{i, 1}, w_{i, 2}$ are determined by autonomous EV agents while $C(t)$ and $P_{V 2 G}(t)$ are set by the capacity controller.

In respect to (1) and (2), it can be verified that these equations represent two convex optimization problems [25]. Many numerical methods are able to provide the instantaneous solution, but the dynamic nature of the problem, the geographical spread of the EV population and scalability of solution can present difficulties when centralized solvers are used. We note that (1) and (2) represent two problems that change with time. EVs/non-EV loads arrive and depart at random times. A centralized solver would need to collect data and solve the problem every time some change occurs. Therefore, our interest is in distributed and on-line optimization approaches since recharging sockets need to solve the problem in real time with minimum communication overhead. We choose a solution approach based on solving convex optimization problems using sliding mode control [26] which yields an online distributed solution that requires only binary information about the state of the system which in our case is frequency. Using results from [27], it can be shown that the following differential inclusions converge to the solutions of the problems described by (1) and (2).

\section{Mode 1}

$$
\frac{d\left(p_{i}(t)\right)}{d t}=\frac{\alpha w_{i, 1}(t)}{p_{i}(t)} \overline{\theta_{1}} \overline{\phi_{1}\left(p_{i}(t)\right)}-\beta\left(\theta_{1}+\phi_{1}\left(p_{i}(t)\right)\right)
$$

where

$$
\begin{aligned}
& \theta_{1}= \begin{cases}1 & \text { if } \sum_{i \mid v_{i} \in V(t)} p_{i}(t) \geq C(t), \\
0 & \text { otherwise }\end{cases} \\
& \phi_{1}\left(p_{i}(t)\right)= \begin{cases}1 & \text { if } p_{i}(t) \geq p_{\text {imax }} \\
-1 & \text { if } p_{i}(t) \leq 0 \\
0 & \text { otherwise }\end{cases}
\end{aligned}
$$

and

$$
\overline{\phi_{1}\left(p_{i}(t)\right)}= \begin{cases}1 & \text { if } \phi\left(p_{i}(t)\right)=0, \\ 0 & \text { otherwise }\end{cases}
$$

$\underline{\text { Mode } 2}$

$$
\frac{d\left(p_{i}(t)\right)}{d t}=\frac{\alpha w_{i, 2}(t)}{p_{i}(t)} \overline{\theta_{2}} \overline{\phi_{2}\left(p_{i}(t)\right)}+\beta\left(\theta_{2}+\phi_{2}\left(p_{i}(t)\right)\right)
$$

where

$$
\theta_{2}= \begin{cases}1 & \text { if }-\sum_{i \mid v_{i} \in V_{V 2 G}(t)} p_{i}(t) \geq P_{V 2 G}(t), \\ 0 & \text { otherwise }\end{cases}
$$




$$
\phi_{2}\left(p_{i}(t)\right)= \begin{cases}1 & \text { if } p_{i}(t) \leq-p_{\text {imaxV } 2 G}, \\ -1 & \text { if } p_{i}(t) \geq 0 \\ 0 & \text { otherwise }\end{cases}
$$

Here $\alpha>0$ and $\beta>0$ are tunable parameters which are the same for all recharging sockets.

\section{B. Optimal Recharging Rate}

In Mode 1, at the optimal point of (1) the recharging rate of $i$ th $\mathrm{EV}$ is given by

$$
p_{i}= \begin{cases}\kappa w_{i} & \text { if } \kappa w_{i}<p_{i, \max }, \\ p_{\text {imax }} & \text { otherwise }\end{cases}
$$

where $\kappa$ is some constant which is the same for all EVs and has units of $[k w h / \$]$. We refer to $1 / \kappa$ as price per unit of energy.

Proof: $p_{i} \leq p_{i, \max }$ as this condition is enforced by the second constraint in (1).

To show the recharging rate at the optimal point of the problem in (1), we start by considering all the EVs. let $N_{j}=$ $N, C_{j}=C$ and $V_{j}=V$ and $j=1$ where $j$ indicates the iteration number of the following procedure. Here $S j$ is used as abbreviation of step $\mathrm{j}$.

1) Procedure A:

S1) Assume that second constraint is absent. We solve (1) only with first constraint. Dropping the second constraint, we transform the optimization problem (1) into an equivalent problem by a change of variable.

$$
x_{i}=\log \left(p_{i}\right)
$$

and formulate an equivalent problem (12) to the original problem as follows

$$
\begin{array}{ll}
\underset{x_{i}}{\operatorname{maximize}} & \sum_{i \mid v_{i} \in V_{j}(t)} w_{i, 1}(t) x_{i}(t) \\
\text { subject to } & \sum_{i \mid v_{i} \in V_{j}(t)} e^{x_{i}(t)} \leq C_{j}(t)
\end{array}
$$

Since the objective is linear we can use vector notation to represent it. Let us define $\mathbf{W}_{\mathbf{1}, \mathbf{j}}=$ $\left[w_{1,1}, \ldots, w_{N_{j}, 1}\right]^{T} \in \mathbb{R}^{N_{j}}, \mathbf{X}_{\mathbf{j}}=\left[x_{1}, \ldots, x_{N_{j}}\right]^{T} \in$ $\mathbb{R}^{N_{j}}, \mathbf{E}_{\mathbf{x}, \mathbf{j}}=\left[e^{x_{1}}, \ldots, e^{x_{N_{j}}}\right]^{T}$ and $\mathbf{P}_{\text {max }, \mathbf{j}}=$ $\left[p_{1 \max }, \ldots, p_{N_{j} \max }\right]^{T}$. For simplicity we can drop the notation that shows dependence on $\mathrm{t}$ and write

$$
\begin{array}{ll}
\underset{X_{j}}{\operatorname{maximize}} & \mathbf{W}_{\mathbf{1}, \mathbf{j}}{ }^{T} \mathbf{X}_{\mathbf{j}} \\
\text { subject to } & \mathbf{1}^{T} \mathbf{E}_{\mathbf{x}, \mathbf{j}} \leq C_{j}
\end{array}
$$

Here, we are maximizing a linear function over a convex region. Thus at the optimal point, the hyper plane $\mathbf{W}_{\mathbf{1}}{ }^{T} \mathbf{X}=b$ must be tangent to the feasible region where $b \in \mathbb{R}$. We note that at the optimal point, $\mathbf{W}_{\mathbf{1}, \mathbf{j}}{ }^{T} \mathbf{X}=b$ is tangent to a level curve of function $\mathbf{1}^{T} \mathbf{E}_{\mathbf{x}, \mathbf{j}}$ corresponding to the level curve defined by $\mathbf{1}^{T} \mathbf{E}_{\mathbf{x}, \mathbf{j}}=C_{j}$. Now since the gradient of a function is normal to its level curves, we conclude that $\mathbf{W}_{\mathbf{1 , j}}$ is parallel to the gradient of $\mathbf{1}^{T} E_{x, j}$.
Note that $\nabla\left(\mathbf{1}^{T} \mathbf{E}_{\mathbf{x}, \mathbf{j}}\right)=\mathbf{E}_{\mathbf{x}, \mathbf{j}}=\mathbf{P}_{\mathbf{j}}=\left[p_{1}, \ldots, p_{N_{j}}\right]^{T}$ Thus we can write

$$
\frac{\mathbf{W}_{\mathbf{1}, \mathbf{j}}}{\left\|\mathbf{W}_{\mathbf{1}, \mathbf{j}}\right\|}=\frac{\mathbf{P}_{\mathbf{j}}}{\left\|\mathbf{P}_{\mathbf{j}}\right\|}
$$

where $\|$.$\| is the Euclidean Norm and hence, p_{i}=\kappa_{j} w_{i}$ for $i=1, \ldots, N_{j}$ where $\kappa_{j}=\frac{\left\|\mathbf{W}_{\mathbf{1}, \mathbf{j}}\right\|}{\left\|\mathbf{P}_{\mathbf{j}}\right\|}$

S2) We divide EVs into two sets $G_{1}$ and $G_{2}$ depending on the solution of the most recent iteration of S1. An EV is assigned a set using following equation.

$$
v_{i} \in \begin{cases}G_{1, j} & \text { if } \kappa_{j} w_{i, 1}<p_{i, \max }, \\ G_{2, j} & \text { otherwise }\end{cases}
$$

where all EVs in $G_{2, j}$ are those whose recharging rates would violate the second constraint in (1) if it was present.

S3) If $G_{2, j}$ is empty then go to S4. otherwise, for all EVs in $G_{2, j}$, assign $p_{i}=p_{i, \max }$. For these EVs we have found the optimal solution because the objective is concave increasing in $p_{i}$ and it can be shown that $\kappa_{j+1} \geq \kappa_{j}$ for all $j \geq 1$. For rest of EVs in $G_{1, j}$, if $G_{1, j}$ is empty, then go to S5. Otherwise set $C_{j+1}=C_{j}-\sum_{i \mid v_{i} \in G_{2, j}} p_{i, \max }$, $V_{j+1}=G_{1, j}, N_{j+1}=\left|G_{1, j}\right|$ and go to $\mathrm{S} 1$ with reduced problem under consideration.

S4) Now for all EVs left under consideration, none must be recharging such that the second constraint in (1) can be violated. Hence we have reached the solution. Go to S6.

S5) Since $G_{1, j}$ is empty, we stop because for all EVs $p_{i}=$ $p_{i, \max }$ and go to S6.

S6) Suppose we reach $S 6$ in qth iteration. It follows that we can define $G_{1}=G_{1, q}$ and $G_{2}=\cup_{j=1}^{q} G_{2, j}$ which contains all EVs recharging at maximum possible recharging rate and $\kappa=\kappa_{q}$ where

$$
v_{i} \in \begin{cases}G_{1} & \text { if } p_{i}=\kappa w_{i, 1}<p_{i, \max } \\ G_{2} & \text { if } p_{i}=p_{i, \max }\end{cases}
$$

Since a given EV in $V(t)$ must be either in $G_{1}$ or in $G_{2}$, the equation (10) follows.

The EVs in $G_{1}$ are the recipients of the proportional service because their recharging rates are directly proportional to their respective payment rates. We consider EVs in $G_{2}$ as the recipients of the priority service since they are recharged at the maximum possible recharging rate.

\section{Value of $\kappa$ in $j$ th Iteration of Procedure A}

During $j$ th iteration of step 1 of procedure A,

$$
p_{i}=\frac{w_{i, 1}}{\sum_{k=1}^{N_{j}} w_{k, 1}} C_{j}
$$

Proof: Here we show that

$$
p_{i}=\frac{w_{i, 1}}{\sum_{k=1}^{N_{j}} w_{k, 1}} C_{j} \Leftrightarrow p_{i}=w_{i, 1} \frac{\left\|\mathbf{P}_{\mathbf{j}}\right\|}{\left\|\mathbf{W}_{\mathbf{1}, \mathbf{j}}\right\|}
$$


suppose

$$
p_{i}=\frac{w_{i, 1}}{\sum_{k=1}^{N_{j}} w_{k, 1}} C_{j}
$$

then

$$
p_{i}=\frac{w_{i, 1}}{\sum_{k=1}^{N_{j}} w_{k, 1}} \frac{\left\|\mathbf{W}_{\mathbf{1}, \mathbf{j}}\right\|}{\left\|\mathbf{W}_{\mathbf{1}, \mathbf{j}}\right\|} C_{j}
$$

thus

$$
p_{i}=\frac{w_{i, 1}}{\left\|\mathbf{W}_{\mathbf{1}, \mathbf{j}}\right\|} \sqrt{\left(\frac{w_{1,1} C_{j}}{\sum_{k=1}^{N_{j}} w_{k, 1}}\right)^{2}+\cdots+\left(\frac{w_{N_{j}, 1} C_{j}}{\sum_{k=1}^{N_{j}} w_{k, 1}}\right)^{2}}
$$

hence

$$
p_{i}=\frac{w_{i, 1}}{\left\|\mathbf{W}_{\mathbf{1}, \mathbf{j}}\right\|} \sqrt{p_{1}^{2}+\cdots+p_{N_{j}}^{2}}
$$

thus

$$
p_{i}=w_{i, 1} \frac{\left\|\mathbf{P}_{\mathbf{j}}\right\|}{\left\|\mathbf{W}_{\mathbf{1}, \mathbf{j}}\right\|}
$$

alternatively suppose

$$
p_{i}=w_{i, 1} \frac{\left\|\mathbf{P}_{\mathbf{j}}\right\|}{\left\|\mathbf{W}_{\mathbf{1}, \mathbf{j}}\right\|} \text { for } i=1 \ldots N_{j}
$$

then

$$
\sum_{k=1}^{N_{j}} p_{k}=\sum_{k=1}^{N_{j}} w_{k, 1} \frac{\left\|\mathbf{P}_{\mathbf{j}}\right\|}{\left\|\mathbf{W}_{\mathbf{1}, \mathbf{j}}\right\|}
$$

hence

$$
\frac{\left\|\mathbf{P}_{\mathbf{j}}\right\|}{\left\|\mathbf{W}_{\mathbf{1}, \mathbf{j}}\right\|}=\frac{\sum_{k=1}^{N_{j}} p_{k}}{\sum_{k=1}^{N_{j}} w_{k, 1}}
$$

Using this value in (19), we get

$$
p_{i}=w_{i, 1} \frac{\sum_{k=1}^{N_{j}} p_{k}}{\sum_{k=1}^{N_{j}} w_{k, 1}}
$$

but $\sum_{j=1}^{N_{j}} p_{j}=C_{j}$ at the optimal point since objective is concave increasing in $p_{i}$

$$
p_{i}=\frac{w_{i, 1}}{\sum_{k=1}^{N_{j}} w_{k, 1}} C_{j}
$$

Which completes the proof.

Note that this result is true for qth iteration and hence true for every $\mathrm{EV}$ in $G_{1}$ and allows us to view the recharging rate controller as a discriminatory scheduler. Thus for EVs in $G_{1}$ we can write

$$
p_{i}=\frac{w_{i, 1}}{\sum_{k \mid v_{k} \in G_{1}} w_{k, 1}} C_{G_{1}}
$$

and

$$
\kappa=\frac{C_{G_{1}}}{\sum_{k \mid v_{k} \in G_{1}} w_{k, 1}}
$$

where $C_{G_{1}}$ is capacity being used to recharge EVs in $G_{1}$.

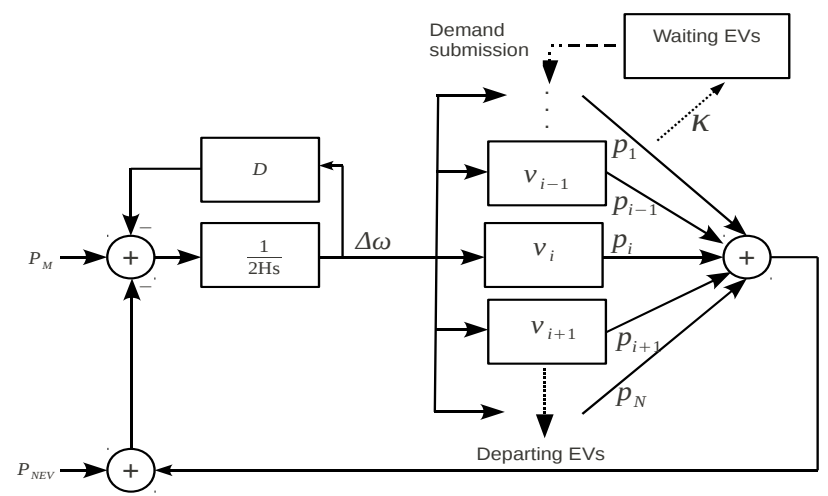

Fig. 2. Implementation using a single turbine and isolated synchronous machine

\section{Characteristics of $\kappa$}

Using the proposed recharging rate control, autonomous EV agents are encouraged to submit demand at the time of high availability of energy and to disperse their demand relative to each other. From (21), the following characteristics of the incentive policy $\kappa$ can be identified.

1) $\kappa$ is directly proportional to the available generation capacity. For a given payment rate, EVs get better recharging rates if their demand time matches with the time of high availability.

2) $\kappa$ is a monotonically decreasing function of the number of recharging EVs. When large number of EVs submit demand at the same time, the recharging rates reduce for all EVs. For a given payment rate, EVs can achieve better recharging rates if they disperse their demand relative to each other instead of submitting it at the same time.

\section{E. Pareto Efficiency}

Let $u_{i}\left(p_{i}, w_{i, 1}\right)$ be the utility of ith $\mathrm{EV}$ which is concave increasing in $p_{i}$ and decreasing in $w_{i, 1}$. Using the results presented in [28], it can be shown that, assuming that all EVs are rational and autonomous, and that they observe the same value of $\kappa$ for their chosen $w_{i, 1}$, the equilibrium recharging rates are Pareto efficient.

\section{F. Proportional Fairness}

Let $\mathbf{P}_{\mathbf{0}}=\left[p_{01}, p_{02}, \ldots, p_{0 N}\right]$ be a recharging rate vector for $\mathrm{N}$ EVs that is feasible. For a given payment rate vector $\mathbf{W}_{\mathbf{1}}=$ $\left[w_{1,1}, w_{2,1}, \ldots, w_{N, 1}\right]$, we say that $\mathbf{P}_{\mathbf{0}}$ is proportionally fair if for any other feasible $\mathbf{P}_{\mathbf{1}} \neq \mathbf{P}_{\mathbf{0}}$ the aggregate weighted proportional change is negative. i.e.,

$$
\sum_{i=1}^{N} w_{0 i} \frac{p_{1 i}-p_{0 i}}{p_{0 i}}<0
$$

Using a similar argument as presented in [22], it can be shown that the optimal recharging rates are proportionally fair. 


\section{IMPLEMENTATION}

\section{A. Single Machine Implementation}

We first consider an isolated and unregulated synchronous machine who's rotor is initially rotating at synchronous speed and arbitrary initial EV recharging rates such that initial net torque on rotor is zero. Such a machine can be modelled by the following linear differential equation [29]

$$
2 H \frac{d(\Delta \omega(t))}{d t}+D \Delta \omega(t)=P_{M}(t)-P_{N E V}(t)-\sum_{i=1}^{N} p_{i}(t)
$$

Where $\mathrm{H}[\mathrm{p} . \mathrm{u}]$ is the inertia constant of machine, $D$ [p.u] is the damping torque, $\Delta \omega[\mathrm{p} . \mathrm{u}]$ is the frequency deviation and $P_{M}$ [p.u] is the input mechanical torque to the machine which is the scheduled power.

Let $0 \leq P_{M} \leq 1,0<D \leq 1, H>0$ and $0<P_{N E V} \leq$ 1 be constants such that $P_{M}-P_{N E V}<\sum_{i=1}^{N} p_{\text {imax }}$ and $P_{M}-P_{N E V}-\sum_{i=1}^{N} p_{i}(0) \approx 0$ then from any given initial values of $0<p_{i}(0) \leq p_{\text {imax }}$ for $i=1, \ldots N$, the system of differential inclusions (24) converges to $p_{i}(t)$ as in (10) with $\kappa$ as in (21) such that $|\Delta \omega| \leq \Delta \zeta$ where $\Delta \zeta$ is small as compared to the statutory frequency deviation.

$2 H \frac{d(\Delta \omega(t))}{d t}+D \Delta \omega(t)-P_{M}(t)+\sum_{j=1}^{N} p_{j}(t)+P_{N E V}(t)=0$ $\frac{d\left(p_{i}(t)\right)}{d t}-\frac{\alpha w_{i, 1}(t)}{p_{i}(t)} \overline{\theta_{1}(t)} \overline{\phi_{1}\left(p_{i}(t)\right)}-\beta\left(\theta_{1}(t)+\phi_{1}\left(p_{i}(t)\right)\right)=0$ for $i=1,2, \ldots, \mathrm{N}$

Computing the values of $\theta_{1}$ and $\theta_{2}$ using equations (4) and (8) will require knowledge of recharging rates $p_{i}$ of all EVs. To ensure scalability, we compute the value of $\theta_{1}$ and $\theta_{2}$ by using frequency deviation $\Delta \omega$.

$$
\theta_{1}= \begin{cases}1 & \text { if } \Delta \omega<0 \\ 0 & \text { otherwise }\end{cases}
$$

and

$$
\theta_{2}= \begin{cases}0 & \text { if } \Delta \omega<\Delta \omega_{V 2 G} \\ 1 & \text { otherwise }\end{cases}
$$

Proof:

The first equation in (24) can be rewritten as

$$
2 H \frac{d(\Delta \omega(t))}{d t}+D \Delta \omega(t)=C(t)-P_{E V}(t)
$$

where $C(t)=P_{M}(t)-P_{N E V}(t)$ and $P_{E V}(t)=$ $\sum_{j=1}^{N} p_{j}(t)$

Dividing both sides (27) by $2 H$, multiplying by integrating factor $e^{\left(\frac{D}{2 H}\right) t}$, integrating and multiplying by $e^{-\left(\frac{D}{2 H}\right) t}$ after integration, we get

$$
\begin{aligned}
& \Delta \omega(t)= \\
& \quad e^{-\left(\frac{D}{2 H}\right) t}\left[\int_{0}^{t} \frac{e^{\left(\frac{D}{2 H}\right) \tau}}{2 H}\left(C(\tau)-P_{E V}(\tau)\right) d \tau+c_{0}\right]
\end{aligned}
$$

We can write

$$
\Delta \omega(t)=g_{1}(t) g_{2}(t)
$$

where $g_{1}(t)=e^{-\left(\frac{D}{2 H}\right) t}$ and

$$
g_{2}(t)=\left[\int_{0}^{t} \frac{e^{\left(\frac{D}{2 H}\right) \tau}}{2 H}\left(C(\tau)-P_{E V}(\tau)\right) d \tau+c_{0}\right]
$$

If $\theta_{1}=0$ :

Suppose $0<\Delta \omega(0)=c_{0}<\Delta \zeta$, we take $\theta_{1}=0$. Hence $p_{j}(t)=\min \left(\int_{0}^{t}\left(\frac{\alpha w_{i}(t)}{p_{j}(t)} d t\right), p_{j, \max }\right)$. Hence $P_{E V}(t)=$ $\sum_{j=1}^{N} \min \left(\int_{0}^{t}\left(\frac{\alpha w_{i}(t)}{p_{j}(t)} d t\right), p_{j, \max }\right)$ is a monotonically increasing function of time. Since $C(t)<\sum_{i=1}^{N} p_{\text {imax }}$, it follows that $C(t)<P_{E V}(t)$ for some $t>t_{0}$. If $C(0)<$ $P_{E V}(0)$, then $t_{0}=0$. It follows from (30), that $g_{2}(t)$ increases monotonically for $t<t_{0}$ and starts decreasing for $t>t_{0}$. This decrease is rapid because $e^{\frac{D}{2 H} t}$ increase rapidly although $C(t)-P_{E V}(t)<0$ is very small. Thus we conclude that $\Delta \omega(t)<0$ for some $t>t_{1}>t_{0}$ and hence $\theta_{1}=1$ for $t>t_{1}$. If $\theta_{1}=1$ :

Now, suppose $-\Delta \zeta<\Delta \omega(0)=c_{0}<0$, we take $\theta_{1}=1$. Hence $p_{j}=\max \left(p_{j}(0)-\beta t, 0\right)$. Here $P_{E V}(t)=$ $\sum_{j=1}^{N} \max \left(p_{j}(0)-\beta t, 0\right)$ is a monotonically decreasing function of time. Since $C(t)>0$, it follows that $C(t)>P_{E V}(t)$ for some $t>t_{3}$. If $C(0)>P_{E V}(0)$, then $t_{3}=0$. It follows from equation (30), that $g_{2}(t)$ decreases for $t<t_{3}$ and then increases for $t>t_{3}$. Hence $\Delta \omega(t)>0$ for some $t>t_{4}>t_{3}$.

Hence the equilibrium point of (24) is the same as the equilibrium point of (3).

In respect to the previous proof, we note that the frequency deviation follows a differential equation and lags the imbalance between generation and demand as it is shown by (28). This frequency deviation is used to create a sliding mode at $\Delta \omega(t)=0$, which also creates a corresponding sliding mode at $C(t)=P_{E V}(t)$.

We also note that it is possible to use frequency deviation $\Delta \omega(t)$ as proxy to compute $\theta_{1}$ instead of using equation (4). Thus, the recharging rates that converge to (10) can be calculated in a distributed manner.

We note that alternative methods for extracting the required information on power imbalance may exist but we leave this line of investigation for future research.

Figure 3 shows the recharging rates $p_{i}$ and frequency deviation $\Delta \omega$ when recharging 5 EVs using $P_{\text {base }}=100[\mathrm{kw}]$ generator. Here, $H=1$ [s], $D=1$ [p.u], $P_{N E V}=0.9$ [p.u], $\sum_{i=1}^{N} p_{i}=0.1$ [p.u] with random $p_{i}, \mathbf{W}_{\mathbf{1}}=[1,2,3,4,5]$ $\alpha=1, \beta=1$. It can be observed that if total load is initially balanced then the recharging rate controller achieves intra EV redistribution of recharging rates such that the recharging rates converge to $\mathbf{P}=[0.67,1.33,2,2.67,3.33][\mathrm{kw}]$ which are in accordance with their respective payment rates. The redistribution is achieved in such a way that the rotor of the synchronous machine rotates at synchronous speed and hence a change in input torque is not required and $1 / \kappa$ converges to $0.67[\mathrm{kwh} / \$]$ at all the recharging sockets.

We note that the following are special cases when an intraEV redistribution of recharging rates will occur 


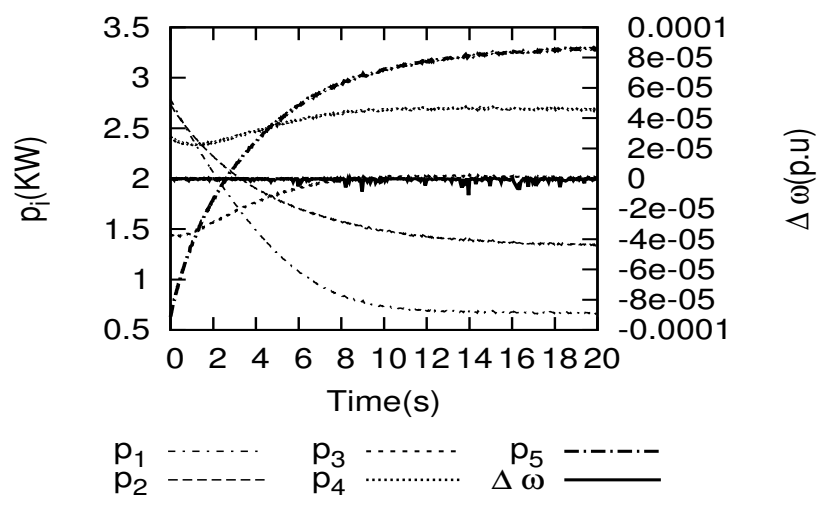

Fig. 3. Recharging rates and frequency deviation for single machine case

i) Demand submitted by a new EV

ii) Completion of a demand or departure of an EV

iii) A change in $w_{i, 1}$ by ith $\mathrm{EV}$ agent.

iv) A change in Non-EV load

\section{B. EV Agent's Budget and Decentralized Billing}

When EV agents connect to the socket they know the budget $B d$ that they have at their disposal to achieve, e.g., a target battery state of charge $B S O C_{i}$ before their next journey. What they do not know is the state of the electric grid and hence they are unaware of the value of $1 / \kappa$. However, all EV agents can submit a small $w_{i, 1}$ and within a few seconds (Section V-E, Fig. 5) they will receive from the socket agent the signal $1 / \kappa$. From this point onwards the EV agent can start making informed decisions by tracking its remaining budget and estimating the total cost of recharging: as a first approximation at the moment of connection $B d \geq \overline{M P_{i}} \approx w_{i, 1} * T_{L}$ where $T_{L}=t_{1}-t_{0}$ is the total time available to recharge the battery. Here, $t_{0}$ is the EV initial time of connection to socket and $t_{1}$ is the time of departure of the EV. Therefore, the EV agent and the sockets agent can estimate the cost incurred (current value of bill) using (31).

$$
M P_{i}\left(t_{n}\right)=\int_{t_{0}}^{t_{n}} w_{i, 1}(t) d t
$$

Where $t_{n}$ is the current time. The EV agent will also be able to monitor at all times its state of recharge using (32):

$$
\Delta B S O C_{i}\left(t_{0}, t_{n}\right)=\int_{t_{0}}^{t_{n}} p_{i}(t) d t
$$

Hence, each EV agent can continuously monitor the changes in $1 / \kappa$ and dynamically adapt the value of $w_{i, j}$ to satisfy their own internal objectives. For example, it could be constantly aiming at saving as much as possible from its remaining budget $\left(B d-M P_{i}\left(t_{n}\right) \geq 0\right)$ as long its target $B S O C_{i}\left(t_{n}\right)$ is being achieved.

A rational EV agent will stop paying once its battery has been recharged to the target capacity. Hence the EV agent can set $w_{i, 1}=0$ and this will instantly stop recharging the EV battery. At the time of disconnection, the socket agent will have no further information on $w_{i, 1}$ and the bill can be transmitted to a centralized location.

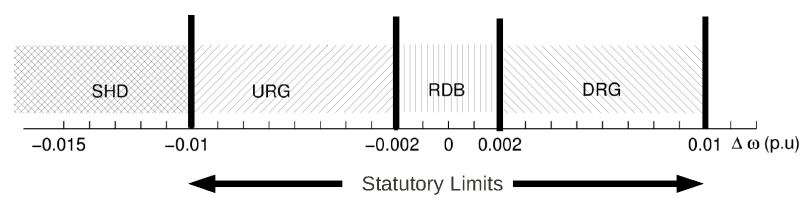

Fig. 4. Frequency to function map for the socket manager

\section{A Protocol Based on the Recharging Rate Controller}

In order to deploy the recharging rate controller in a multimachine system, we will need to take into account the following considerations.

1) Local frequency measurement estimates are based on voltages and currents at the sockets which will contain various harmonics due to intra machine rotor oscillations, distortion and noise. It might not be possible to measure frequency to the desired accuracy with high resolution at each socket.

2) Different sections of network may have slight mismatch in frequency at a given instant in time. The measurements taken by all sockets are not necessarily the same.

Therefore, we modify the recharging rate controller and propose a socket management protocol. We consider a socket manager agent that can communicate with the sockets and other controllers in the power system. The socket manager broadcasts a pseudo frequency signal to all sockets. Two messages are sufficient to send the pseudo frequency signal.

i) FAN frequency above nominal

ii) FBN frequency below nominal

In addition the socket manager broadcasts two messages that are not part of the original control. These messages can temporarily hold the socket state or shed its load when needed.

iii) LSS lock socket state

iv) SSL shed socket load

let $m\left(t_{k}\right)$ be a message received by ith socket at time $t_{k}$. The recharging rate for ith socket is given by (33) on page 9 , where $\Delta \gamma$ is an arbitrarily small constant.

The socket manager has the following key functions.

1) Generates the pseudo frequency signal for recharging rate controllers

2) Allows any critical control to act without interference from recharging rate controllers by allowing locking of sockets.

3) Integrates with the protection system to shed all EV load or sheds all EV load if frequency falls below the statutory limit and switches the sockets to Mode 2. Those EVs which are willing to discharge are used as V2G sources.

To generate the pseudo frequency the socket manager maps the frequency to functions as shown in Fig. 4 where the functions, their descriptions and the corresponding messages are shown in the following text.

1) SHD sheds all EV load. A SSL messages is transmitted every $\mathrm{T}[\mathrm{ms}]$.

2) URG Decreases the recharging capacity for EVs. A FBN message is transmitted every $\mathrm{T}[\mathrm{ms}]$. 


$$
p_{i}\left(t_{k} \leq t \leq t_{k+1}\right)= \begin{cases}p_{i}\left(t_{k}\right)+\int_{t_{k}}^{t}\left(\frac{w_{i, 1} \alpha}{p_{i}(\tau)}\right) d \tau-\beta \phi\left(p_{i}(t)\right)\left(t-t_{k}\right) & \text { if } m\left(t_{k}\right)=F A N \text { and } p_{i}\left(t_{k}\right) \neq 0 \\ \Delta \gamma & \text { if } m\left(t_{k}\right)=F A N \text { and } p_{i}\left(t_{k}\right)=0 \\ p_{i}\left(t_{k}\right)-\beta\left(1+\phi\left(p_{i}(t)\right)\right)\left(t-t_{k}\right) & \text { if } m\left(t_{k}\right)=F B N \\ 0 & \text { if } m\left(t_{k}\right)=S S L \\ p_{i}\left(t_{k}\right) & \text { if } m\left(t_{k}\right)=L S S \text { or message loss in transit }\end{cases}
$$

3) RDB Redistributes recharging rates among EVs according to their respective payment rates. A FAN message is transmitted and a FBN message follows it by $T_{f}[\mathrm{~ms}]$ where $T_{f} \leq T$. The pattern is repeated every $\mathrm{T}[\mathrm{ms}]$. $T_{f}$ is computed such that $T_{f}=0$ at the lower boundary of RDB region of map in Fig. 4 and increases linearly to $T_{f}=T$ at the upper boundary of RDB region.

4) DRG Increase the capacity for recharging EVs. A FAN message is transmitted very $\mathrm{T}[\mathrm{ms}]$.

\section{Benefits of Proposed Protocol}

1) All sockets act on the same information and do not need to accurately measure the frequency with high resolution.

2) Message communication to all sockets is broadcast, hence, addressing of individual sockets is not needed.

3) Recharging sockets can temporarily use local frequency measurements if communication link is broken and can revert to using global measurement when link is reestablished

4) Exact measurement of frequency is not required at each socket and pseudo frequency can be generated using an approximation of exact frequency

5) Interference to critical controls in the power system is avoided by locking sockets when needed.

\section{E. Simulation Results}

In this section we present simulation results with the recharging rate control implemented together with the socket management protocol. We use a power system with primary frequency regulation provided by steam reheat turbines with droop based governors as modelled in [30]. The average frequency deviation $\Delta \omega$ can be expressed by the following equation as derived in [30].

$\Delta \omega=\left(\frac{R \omega_{n}^{2}}{D R+1}\right)\left(\frac{\left(1+F_{H} T_{R}\right) P_{S P}-\left(1+T_{R} s\right) P_{e}}{s^{2}+2 \zeta \omega_{n} s+\omega_{n}^{2}}\right)$

Where

$$
\begin{gathered}
\omega_{n}^{2}=\frac{D R+1}{2 H R T_{R}} \\
\zeta=\omega_{n}\left(\frac{2 H R+\left(D R+F_{H}\right) T_{R}}{2 D_{R}+1}\right)
\end{gathered}
$$

and

$$
P_{e}=P_{S C H}-P_{N E V}-P_{E V}
$$

Where

$R=$ Speed droop or regulation,

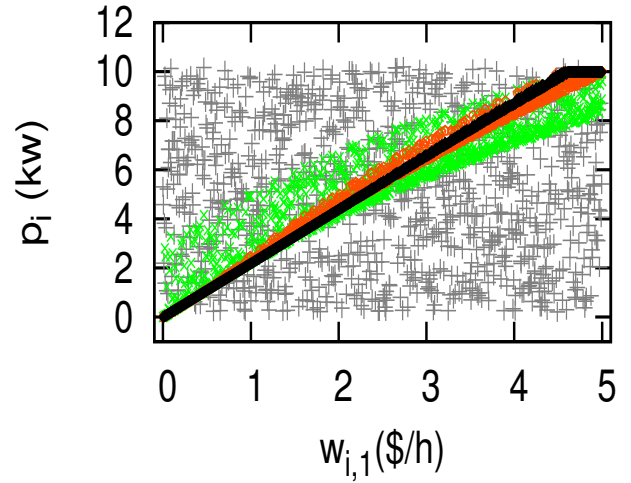

$\mathrm{t}=0 \mathrm{~s}$ $25 \mathrm{~s}$ $50 \mathrm{~s}$ $100 \mathrm{~s}$

Fig. 5. Snapshot of recharging rates as function of $w_{i, 1}$

$F_{H}=$ Fraction of power from high pressure section of turbine,

$T_{R}=$ Reheat time constant $[\mathrm{s}]$,

$P_{e}=$ Electrical power [p.u],

$P_{S P}=$ Incremental set point $[\mathrm{p} . \mathrm{u}]$,

The configuration parameters are: $N=1000, P_{\text {base }}=50$ $[\mathrm{Mw}] \alpha=1, \beta=1, P_{N E V}\left(t_{0}\right)=0.9, P_{M}\left(t_{0}\right)=0, t_{0}=0$, $w_{i}$ are uniformly distributed random variables between 0 and $5[\$ / \mathrm{h}]$ (considered constant during the simulation), $p_{i}(t=0)$ are uniformly distributed between 0 and $10[\mathrm{kw}]$ such that $\sum_{i=1}^{N} p_{i}=0.1$ [p.u] (to ensure initial balance condition), $p_{\text {imax }}=10[\mathrm{kw}] H=3.5[\mathrm{~s}], R=0.05, F_{H}=0.3, D=1$, $T_{R}=8[\mathrm{~s}], P_{S P}=0, T=20[\mathrm{~ms}]$ and $R D B$ region has boundaries at $\Delta \omega= \pm 0.0002$ [p.u]

Figure 5 shows the snapshots of recharging rates $p_{i}$ as function of $w_{i}$ at $\mathrm{t}=0,25,50$, and 100 [s] respectively. Thus recharging rates are redistributed and EVs are dynamically partitioned into $G_{1}$ and $G_{2}$ with $\kappa \approx 2.2[\mathrm{kwh} / \$$ ].

Figure 6 shows the frequency deviation for a $1 \%$ sudden non-EV load loss at $\mathrm{t}=0$ [s]. This step change in $C(t)$ contains a wide spectrum of frequencies. When the high frequency components of the step $C(t)$ are significant, the controller detects a significant difference between $P_{E V}(t)$ and $C(t)$ which is reflected in the values of $\Delta \omega$ when $(t \leq 10$ [s]). When $t>10$ [s] the magnitude of high frequency components of $C(t)$ become negligible compared to its moving average value over a few minutes and $P_{E V}(t)$ follows $C(t)$. In the case of this example, it can be seen that, when the proposed recharging rate control is used, the peak frequency deviation is reduced by $50 \%$. Hence, a $66 \%$ smaller primary frequency regulating turbine is sufficient to provide frequency regulation. We note that the highlighted characteristics can be very helpful 


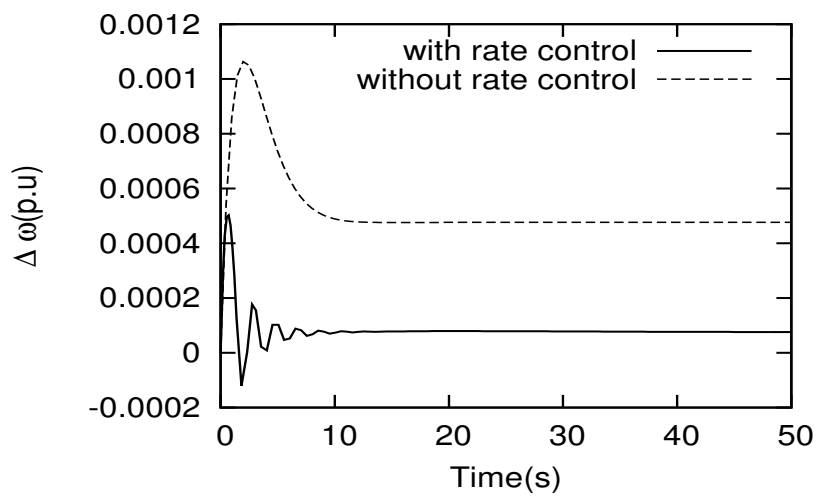

Fig. 6. Frequency deviation with and without proposed recharging rate control

in reducing the turbine size that is needed to regulate the fluctuations of, e.g., the output of renewable energy sources.

For the multiple machine case, we can observe that if the size of $R D B>0$ (Fig. 4) this will result in a steady state value $|\Delta \omega|>0$ which is constant (Fig. 6) and bounded by half of the width of the $R D B$ band. We note that as the width of the $R D B$ region approaches zero, the controller in (33) will in the limit behave as the controller used in the single machine case (24) except in rare circumstances when the EV load is shed because of the frequency falling below the statutory limit.

Since steady state frequency deviation is constant, we observe that $C(t)=P_{E V}(t)$ but a small power will flow from generators providing droop based regulation. However, we would like to point out that had the parked EVs not been present, a much larger power flow from regulating generators would have occurred as observed in Fig. 6.

\section{Recharging Strategies, Demand Deferment AND CAPACITY CONTROL}

As previously mentioned, the socket agents will broadcast the same value of $1 / \kappa$. Assuming that EV agents are perfectly rational and autonomous with utility $u_{i}\left(p_{i}, w_{i, 1}\right)$ such that each EV can exchange $p_{i}=\kappa w_{i, 1}[\mathrm{kw}]$ with $w_{i, 1}[\$ / \mathrm{s}]$ in an environment where the energy providers are maximizing their revenue, the EV agents will choose $w_{i, 1}$ such that the resulting equilibrium recharging rates are Pareto efficient and proportionally fair.

Let $C C R(C)[\$ / h]$ be the generation cost rate of $C[\mathrm{kw}]$. In general, the cost rate is a convex function and the marginal cost rate of generation $\frac{\partial C C R}{\partial C}$ is increasing function of $C$. Thus in the context of a competitive generation market, a generator will maximize revenue if:

$$
\left.\frac{\partial C C R}{\partial C}\right|_{C=P_{N E V}+C_{0}}=\frac{1}{\kappa}=\frac{\sum_{i \mid v_{i} \in G_{1}} w_{i, 1}}{C_{0}}
$$

To show why $\kappa$ encourages deferment of demand by some EVs, we will focus on Mode 1 of operations and we will illustrate a simple game. Let us consider rational and selfish EV agents which try to maximize their BSOC and minimize their total incurred cost. We note that all rational EVs will be in $G_{1}$ as any $\mathrm{EV}$ in $G_{2}$ can decrease its payment rate without decreasing its recharging rate. As we have previously introduced, the total cost incurred by the ith $\mathrm{EV}$ agent over a time $t_{n}-t_{0}$ will be $M P_{i}\left(t_{n}\right)$ (31), and the corresponding change in battery state of charge $\triangle B S O C_{i}\left(t_{n}\right)$ (32). From Fig. 5, we can see that $p_{i}$ converges to $\kappa w_{i, 1}$ in a few seconds even when EVs submit random values of $w_{i, 1}$ at the same time, hence. ${ }^{2}$

$$
\Delta B S O C_{i}\left(t_{1}\right)=\int_{t_{0}}^{t_{1}} p_{i}(t) d t \approx \int_{t_{0}}^{t_{1}} \kappa(t) w_{i, 1}(t) d t
$$

Lets now consider a game with two EVs: $V_{1}, V_{2}$ and two time slots $t_{1}$ and $t_{2}$ of same time width $T_{c}$. We denote a recharging strategy as $\left(t_{i}, t_{j}\right)$ where $V_{1}$ chooses the $i t h$ time slot and $V_{2}$ chooses the $j$ th time slot. In this game, there are four recharging strategies namely $a_{1}=\left(t_{1}, t_{1}\right), a_{2}=$ $\left(t_{1}, t_{2}\right), a_{3}=\left(t_{2}, t_{1}\right)$ and $a_{4}=\left(t_{2}, t_{2}\right)$. For simplicity, we assume that $P_{N E V}$ is the same in both time slots. Also let the equilibrium value of $\kappa$ be $\kappa_{A}$ when EVs choose the same time slot, and $\kappa_{B}$ when they choose different time slots. We define the payoffs of EVs as the ratio $\triangle B S O C\left(t_{1}\right) / M P_{i}\left(t_{1}\right)$ which, in a steady system, as $w_{i, 1}$ is not changing, is the value $\kappa$. We will now show that $a_{1}$ and $a_{4}$ are unstable and $a_{2}$ and $a_{3}$ are pure strategy Nash equilibria. The payoff matrix can be written as

\begin{tabular}{l|cr}
$V_{1}, V_{2}$ & $t_{1}$ & $t_{2}$ \\
\hline$t_{1}$ & $\kappa_{A}, \kappa_{A}$ & $\kappa_{B}, \kappa_{B}$ \\
$t_{2}$ & $\kappa_{B}, \kappa_{B}$ & $\kappa_{A}, \kappa_{A}$
\end{tabular}

TABLE I

PAYOFF MATRIX FOR TWO EVS

When the EVs choose the same time slots, the collective revenue rate of the electric generator from recharging both EVs at the same time is $2 w$ and it will be $w$ when EVs choose different time slots. Let $C_{A}$ denote the capacity allocated by the generator when EVs choose the same time slots and $C_{B}$ denote the capacity allocated by generator when EVs choose different time slots. $C$ is zero in a time slot if no EV chooses that slot. For slots chosen by at least one EV, we now determine $C_{A}$ and $C_{B}$. Thus we can write

$$
\begin{aligned}
& \left.\frac{\partial C C R}{\partial C}\right|_{C=P_{N E V}+C_{A}}=\frac{2 w}{C_{A}} \\
& \left.\frac{\partial C C R}{\partial C}\right|_{C=P_{N E V}+C_{B}}=\frac{w}{C_{B}}
\end{aligned}
$$

Multiplying (41) by 2, rearranging and comparing with a rearranged version of (40), we get

$$
\left.\frac{\partial C C R}{\partial C}\right|_{C=P_{N E V}+C_{A}} C_{A}=\left.2 \frac{\partial C C R}{\partial C}\right|_{C=P_{N E V}+C_{B}} C_{B}
$$

All quantities in (42) are positive. Furthermore, $\frac{\partial C C R}{\partial C}$ is a convex increasing function of $C$. It follows that $C_{A}>C_{B}$ and

$$
\left.\frac{\partial C C R}{\partial C}\right|_{C=P_{N E V}+C_{A}}>\left.\frac{\partial C C R}{\partial C}\right|_{C=P_{N E V}+C_{B}}
$$

${ }^{2} p_{i}$ will converge to $\kappa w_{i}$ even faster than the rate of convergence shown in Fig. 5 when the number of arriving/departing EVs are small compared to the number of already parked EVs. 
Now rearranging (42) we get

$$
C_{A}=2 \frac{\left.\frac{\partial C C R}{\partial C}\right|_{C=P_{N E V}+C_{B}}}{\left.\frac{\partial C C R}{\partial C}\right|_{C=P_{N E V}+C_{A}}} C_{B}
$$

From (44) and (43), we conclude that $C_{A}<2 C_{B}$. Noting that $\frac{1}{\kappa_{A}}=\frac{2 w}{C_{A}}$ and $\frac{1}{\kappa_{B}}=\frac{w}{C_{B}}$, we can write

$$
\kappa_{A}=\kappa_{B} \frac{C_{A}}{2 C_{B}}
$$

and hence,

$$
\kappa_{A}<\kappa_{B}
$$

Using (46), we can interpret the payoff matrix of the game in Table I and observe that $a_{1}$ and $a_{4}$ are unstable, and that $a_{2}$ and $a_{3}$ are two pure Nash equilibria strategies. Hence both EVs will benefit if only one of them defers its demand. Furthermore, we note that there are an unlimited number of equivalent strategies as any $\mathrm{EV}$ does not need to recharge continuously in one session.

If we consider EVs as players in this iterative game, each EV agent will endeavour to achieve, e.g., a desired $\triangle B S O C\left(t_{n}\right)$ before it's next journey commences while minimizing the total cost incurred. These EV agents are hence allowed to make arbitrary learning moves in real time to experiment without compromising the load balance as socket agents will ensure that policy $\kappa$ is complied at all times. ${ }^{3}$

There is a vast amount of technical literature on game theory [19] which is at the core of the solution to the game highlighted in this section. For example in [31], and in the context of multi-agent learning framework, the use of game theory [19] and reinforcement learning [32] was investigated. In [31] the authors study how to learn to play a Pareto-optimal Nash equilibrium when there exist multiple equilibria and agents may have different preferences. It is beyond the scope of this paper to further expand on more elaborated aspects of this game and its solution. This is left for further research.

\section{Final Remarks And Future Work}

We have proposed a distributed recharging rate control algorithm which combines the objectives of regulating frequency and improving utilization of generators by creating an incentive policy for autonomous EV that are randomly connecting to and disconnecting from the electric grid. The incentive policy $\kappa$ encourages EVs to demand energy when non-EV demand is low and utilization of electric generators needs to be improved. EVs also act as frequency regulators which can control their participation role by modifying their respective payment rate $w_{i, 1}$ as individual EVs connect and disconnect at arbitrary times.

The proposed recharging rate control algorithm can be used to realize a DM solution to, for example, reconcile EVs energy demand profiles with the output of available energy sources. Furthermore, encouraging results show that the

\footnotetext{
${ }^{3}$ Note that since EV agents will learn their recharging strategies based on the value of $\kappa$, they should restrain from learning when either message SSL or message $\mathbf{L S S}$ is active.
}

proposed recharging rate control algorithm can help decrease the required size of frequency regulating turbines.

We now highlight three aspects of the proposed framework that have not been addressed in this paper. i) The emergent behaviour of the EV agent population as a function of their demand submission strategies when their reward and cost functions are defined, ii) the capacity control for recharging EVs that is to be solved as a revenue management problem, and iii) the quantification of decrease in size of frequency regulating turbines as a function of parked and recharging EVs. The preliminary results presented in this paper suggest that all of the above outstanding aspects are worth further investigation.

\section{ACKNOWLEDGMENTS}

The first author is a Commonwealth Scholar, funded by Department for International Development, U.K. The authors wish to thank the anonymous reviewers for their valuable feedback on the previous versions of this paper.

\section{REFERENCES}

[1] IEA, "CO2 emission from fuel combustion," International Energy Agency, France, Tech. Rep., Dec. 2009.

[2] J. Barkenbus, "Our electric automotive future: $\mathrm{CO} 2$ savings through a disruptive technology," Policy and Society, vol. 27, no. 4, pp. 399-410, Mar. 2009.

[3] R. Green II, L. Wang, and M. Alam, "The impact of plug-in hybrid electric vehicles on distribution networks: A review and outlook," Renewable and Sustainable Energy Reviews, vol. 15, no. 1, pp. 544 - 553, Aug. 2011.

[4] L. Goeransson and F. Johnsson, "Dispatch modeling of a regional power generation system-Integrating wind power," Renewable Energy, vol. 34 , no. 4, pp. 1040-1049, Apr. 2009.

[5] J. Turner, "A realizable renewable energy future," Science, vol. 285, no. 5428, pp. 687-689, Jul. 1999.

[6] W. Kempton and J. Tomić, "Vehicle-to-grid power implementation: From stabilizing the grid to supporting large-scale renewable energy," Journal of Power Sources, vol. 144, no. 1, pp. 280-294, Jun. 2005.

[7] M. Galus and G. Andersson, "Demand management of grid connected plug-in hybrid electric vehicles (PHEV)," in Energy 2030 Conference, 2008. ENERGY 2008. IEEE, Nov. 2008, pp. 1-8.

[8] H. Lund and W. Kempton, "Integration of renewable energy into the transport and electricity sectors through V2G," Energy Policy, vol. 36, no. 9, pp. 3578-3587, Sep. 2008.

[9] W. Kempton and S. Letendre, "Electric vehicles as a new power source for electric utilities," Transportation Research Part D: Transport and Environment, vol. 2, no. 3, pp. 157-175, Sep. 1997.

[10] J. Lopes, F. Soares, and P. Almeida, "Identifying management procedures to deal with connection of electric vehicles in the grid," in PowerTech, 2009 IEEE Bucharest, Jul. 2009, pp. 1 -8.

[11] C. White and K. Zhang, "Using vehicle-to-grid technology for frequency regulation and peak-load reduction," Journal of Power Sources, vol. 196, no. 8, pp. 3972-3980, Apr. 2011

[12] S. Acha, T. C. Green, and N. Shah, "Optimal charging strategies of electric vehicles in the UK power market," in Innovative Smart Grid Technologies (ISGT), 2011 IEEE PES, Jan. 2011, pp. 1-8.

[13] Z. Ma, D. Callaway, and I. Hiskens, "Optimal charging control for plugin electric vehicles," in Control and Optimization Methods for Electric Smart Grids, ser. Power Electronics and Power Systems. Springer US, 2012, vol. 3, pp. 259-273.

[14] L. Gan, U. Topcu, and S. Low, "Optimal decentralized protocol for electric vehicle charging," in Decision and Control and European Control Conference (CDC-ECC), 2011 50th IEEE Conference on, Dec. 2011, pp. $5798-5804$.

[15] P. Vytelingum, T. Voice, S. Ramchurn, A. Rogers, and N. Jennings, "Agent-based micro-storage management for the smart grid," in Proceedings of the 9th International Conference on Autonomous Agents and Multiagent Systems: volume 1, ser. AAMAS '10. Richland, SC: International Foundation for Autonomous Agents and Multiagent Systems, 2010, pp. 39-46. 
[16] C. Wei, H. Hu, Q. Chen, and G. Yang, "Learning Agents for Storage Devices Management in the Smart Grid," in Computational Intelligence and Software Engineering (CiSE), 2010 International Conference on. IEEE, Dec. 2010, pp. 1-4.

[17] Y. Wang, R. Zhou, and C. Wen, "Robust controller design for power system load frequency control ," in Control Applications, 1992., First IEEE Conference on, vol. 2, Sep. 1992, pp. 642-646.

[18] S. Han, S. Han, and K. Sezaki, "Development of an optimal vehicle-togrid Aggregator for frequency regulation," Smart Grid, IEEE Transactions on, vol. 1, no. 1, pp. $65-72$, Jun. 2010.

[19] J. V. Neumann, O. Morgenstern, A. Rubinstein, and H. Kuhn, Theory of games and economic behavior. Princeton Univ Pr, 2007.

[20] C. Touati, E. Altman, and J. Galtier, "Generalized nash bargaining solution for bandwidth allocation," Computer Networks, vol. 50, no. 17, pp. $3242-3263,2006$.

[21] H. Yaïche, R. R. Mazumdar, and C. Rosenberg, "A game theoretic framework for bandwidth allocation and pricing in broadband networks," IEEE/ACM Trans. Netw., vol. 8, no. 5, pp. 667-678, Oct. 2000.

[22] F. Kelly, "Charging and rate control for elastic traffic," European Transactions on Telecommunications, vol. 8, no. 1, pp. 33-37, 1997.

[23] R. M. Salles and J. A. Barria, "Fair and efficient dynamic bandwidth allocation for multi-application networks," Computer Networks, vol. 49, no. 6 , pp. $856-877,2005$.

[24] R. M. Salles and J. A. Barria, "Lexicographic maximin optimisation for fair bandwidth allocation in computer networks," European Journal of Operational Research, vol. 185, no. 2, pp. 778 - 794, 2008.

[25] S. Boyd and L. Vandenberghe, Convex optimization. Cambridge Univ Pr, 2004.

[26] V. Utkin, Sliding modes in control and optimization. Springer-Verlag Berlin, 1992.

[27] M. Glazos, S. Hui, and S. Zak, "Sliding modes in solving convex programming problems," SIAM Journal on Control and Optimization, vol. 36, no. 2, pp. 680-697, Mar. 1998.

[28] K. Arrow, An extension of the basic theorems of classical welfare economics. Cowles Commission for Research in Economics, The University of Chicago, 1952.

[29] P. Anderson and A. Fouad, Power System Control and Stability. Iowa State University Press, Ames IA, 1977.

[30] P. Anderson, J. Farquhar, J. Medalia, A. Chayes, J. Wiesner, M. Raskin, W. Reinicke, L. Haus, W. Wang, S. Tantawi et al., Power system protection. Wiley, 1998.

[31] X. Wang and T. Sandholm, "Learning near-pareto-optimal conventions in polynomial time," in Proceedings of the Annual Conference on Neural Information Processing Systems (NIPS), 2003.

[32] W. Usaha and J. Barria, "Reinforcement learning for resource allocation in leo satellite networks," Systems, Man, and Cybernetics, Part B: Cybernetics, IEEE Transactions on, vol. 37, no. 3, pp. 515-527, 2007.

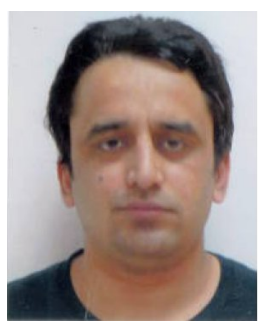

Qazi R. Hamid received his B.E. degree in electrical engineering from University of Engineering and Technology Peshawar, Pakistan, in 2004. He received his M.Sc. degree in communications and signal processing from Imperial College London, U.K., in 2007. He is currently working towards a Ph.D. in electrical engineering with Intelligent Systems and Networks Group, Department of Electrical and Electronic Engineering, Imperial College London. His research interests include multi-agent systems, distributed dynamic resource allocation, power system operations and management of smart grids.

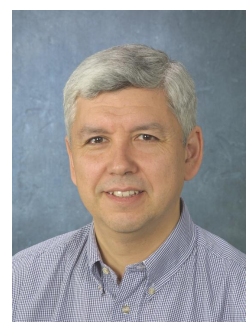

Javier A. Barria (M02) received the Ph.D. degree in electrical and electronic engineering and the M.B.A. degree from the Imperial College London, London, U.K., in 1994 and 1997, respectively. He is currently a Reader with the Intelligent Systems and Networks Group, Department of Electrical and Electronic Engineering, Imperial College London. He has been the joint holder of several European Union Framework and U.K. Engineering and Physical Sciences Research Council project contracts, which are all concerned with aspects of communication systems design and management. His research interests include monitoring strategies for communication and transport networks and distributed resource allocation in dynamic topology networks.

Dr. Barria is a Fellow of the Institution of Engineering and Technology and a Chartered Engineer in the U.K. He was a British Telecom Research Fellow from 2001 to 2002 . 